\title{
ESPECIE NUEVA DE EPIMERIA (AMPHIPODA, EPIMERIIDAE) DEL GOLFO DE PANAMÁ
}

\section{New species of Epimeria (Amphipoda: Epimeriidae) from the Gulf of Panama}

\author{
Carlos Varela
}

Department of Biological Sciences, Biscayne Bay Campus, Florida International University. USA; varela06@gmail.com; (i) orcid.org/0000-0003-3293-7562.

\section{RESUMEN}

Se describe una especie nueva perteneciente al género Epimeria de material colectado durante la expedición del B/I John Elliott Pillsbury en las aguas profundas del Golfo de Panamá. Esta nueva especie puede ser distinguida de las otras especies del género por la combinación de los siguientes caracteres: rostro largo, alcanza la mitad del artejo 2 de la antena 1; ojos ausentes; coxopodito 4 con margen anterovental casi recto dirigido hacia abajo; coxopodito 5 con esquina posteroventral puntiaguda extendiéndose hasta el pleonito 2; pleonitos 5-7 con dientes mediodorsales agudos dirigidos hacia atrás; margen posterior del telson, liso. Este es el primer registro de un representante de la familia Epimeriidae para el Golfo de Panamá.

Palabras clave: Crustacea, Amphipoda, Epimeriidae, Epimeria, aguas profundas, Golfo de Panamá.

\section{ABSTRACT}

A new species of the genus Epimeria is described based on material collected in the expedition of the R/V John Elliott Pillsbury to the deep waters of the Gulf of Panama. This new species could be distinguished of the others in the genus by the combination of several characters including: rostrum long, reaching the half of the second article of the antenna 1; eyes absent; coxopodite 4 with anteroventral margin almost straight down; coxopodite 5 with posteroventral corner elongated backwards, reaching the pleonite 2; pleonites 5-7 with dorsomedial acute teeth directed backwards; posterior margin of the telson, smooth. This is the first record of a member of the family Epimeriidae for the Gulf of Panama.

Keywords: Crustacea, Amphipoda, Epimeriidae, Epimeria, deep waters, Gulf of Panama.

\section{INTRODUCCION}

Los representantes de la familia Epimeriidae exhiben una amplia distribución que abarca prácticamente todos los océanos, habitando ambientes bentónicos fangosos a profundidades de entre 48 y 3710 metros (Winfield et al., 2013). Este taxon es considerado uno de los más emblemáticos del océano austral, donde su riqueza de especies y su diversidad morfológica son mayores que en cualquier otra región marina (d'Udekem d'Acoz y Verheye, 2017). Este género ha sido ampliamente estudiado en los últimos 5 años, en los que se han descrito 38 especies (Coleman y Lowry, 2014; Lörz y Coleman, 2014; Varela y García-Gómez, 2015; Shimomura y Tomikawa, 2016; d'Udekem d'Acoz y Verheye, 2017; Beerman et al., 2018; Verheye et al., 2018). Estudio de material perteneciente a la familia Epimeriidae depositado en el Museo de Invertebrados Marinos de Rosenstiel School of Marine and Atmospheric Science (RSMAS) permitió encontrar una especie del género Epimeria aún no descrita. Esta es la primera cita de la familia Epimeriidae para las aguas del Golfo de Panamá. 


\title{
OBJETIVO
}

- Describir una especie nueva de anfípodo del género Epimeria recolectado en las aguas profundas del Golfo de Panamá, Panamá.

\section{MATERIALES Y MÉTODOS}

El material estudiado fue producto de los viajes de exploración del buque de investigación de la Universidad de Miami, B/I John Elliott Pillsbury en la década de los años 60. Las muestras fueron obtenidas usando una rastra Otter. El ejemplar fue estudiado bajo el microscopio estereoscópico y las ilustraciones se realizaron con un microscopio clínico con cámara lúcida acoplada. Para la terminología y el sistema de clasificación se ha seguido a Lowry y Myers (2017).

El material ha sido depositado en el Museo de Invertebrados Marinos de RSMAS, UMML 32.10270.

\author{
RESULTADOS \\ Taxonomía \\ Orden Amphipoda Latreille, 1816 \\ Suborden Amphilochidea Boeck, 1871 \\ Infraorden Amphilochida Boeck, 1871 \\ Parvorden Amphilochidira Boeck, 1871 \\ Superfamilia Amphilochoidea Boeck, 1871 \\ Familia Epimeriidae Boeck, 1871 \\ Epimeria panamensis sp. nov.
}

(Figs. 1-4)

Diagnosis: Rostro largo, alcanza la mitad del artejo 2 de la antena 1. Ojos ausentes. Coxopodito 4 con margen anteroventral casi recto dirigido hacia debajo. Coxopodito 5 con esquina posteroventral puntiaguda extendiéndose hasta el pleonito 2. Pleonites 5-7 con dientes mediodorsales agudos dirigidos hacia atrás. Margen posterior del telson liso.

Diagnosis (In English): Rostrum long, reaching the half of the second article of the antenna 1. Eyes absent. Coxopodite 4 with anteroventral margin almost straight down. Coxopodito 5 with posteroventral corner elongated backwards, reaching the pleonite 2 . Pleonites 5-7 with dorsomedial acute teeth directed backwards. Posterior margin of the telson, smooth.

Descripción del holotipo. Hembra adulta de $23 \mathrm{~mm}$ de longitud. Región cefálica sinuosa, lóbulo cefálico anterior desarrollado, redondeado y poco proyectado hacia delante. Rostro tan largo como la cabeza, alcanzando casi la mitad del segundo artejo de la antena 1. Ojos ausentes. Pereonito 1 ligeramente más corto que la cabeza, medida sin el rostro, pereonito 2 subigual al pereonito 1, pereonitos del 1 al 4 carecen de procesos medio dorsales o dorsolaterales, pereonitos del 5 al 7 con procesos mediodorsales. Pleonito 1-3 cada uno con un proceso medio dorsal agudo que sobresale del segmento. Urosomito 1 con un proceso medio dorsal puntiagudo, urosomites 2-3 sin procesos medio dorsales (Fig. 1A). Antena 1 (Fig. 1B), artejo 1 del pedúnculo subigual a los artejos 2 y 3 juntos, flagelo accesorio, vestigial, en forma de escama. Antena 2 con el artejo 1 del pedúnculo con un proceso distal, artejo 2 es el más corto, artejo 3 es el más largo con setas en su porción ventral. (Fig. 1C). 
Epistoma más amplio que largo. Labio superior con el margen distal ligeramente bilobulado (Fig. 1D). Labio inferior con los lóbulos internos fusionados, lóbulos externos subtriangulares, proceso mandibular subtriangular (Fig. 1E). Mandíbula derecha con 7 dientes incisivos y lacinia mobilis con 3 dientes (Fig. 1F), mandíbula izquierda con 7 dientes incisivos y lacinia mobilis con 6 dientes (Fig. 1G), molar sin superficie trituradora, margen distal con 10 setas accesorias en la derecha y 12 en la izquierda, palpo mandibular con 3 artejos, artejo 3 subigual al artejo 2. Maxila 1 con el lóbulo interno ovoide, margen distal con 15 setas plumosas, lóbulo externo con su margen distal con 11 setas robustas, el palpo excede la longitud del lóbulo externo, artejo 1 corto, artejo 2 curvado y alargado con robustas setas distales (Fig. 2A). Maxila 2, ambos lóbulos con setas largas en su porción distal, lóbulo externo con setas marginales y grupos de diminutas setas laterales y faciales (Fig. 2B). Maxilípedo con el lóbulo interno que alcanza la mitad de la longitud del lóbulo externo, con 13 robustas setas distales, lóbulo externo ampliamente redondeado distalmente, con siete robustas setas distales. Margen interno y externo de los artejos del palpo con grupos de largas setas, artejo 4 del maxilípedo con pequeños dentículos en su margen interno (Fig. 2C).

Gnatópodo 1 con coxopodito largo y delgado, subagudo ventralmente, basipodito linear, delgado, con el margen ventral irregular en el medio, margen posterior con numerosas setas; isquiopodito corto, con setas distales en el margen posterior; carpopodito ligeramente más corto que el isquiopodito, con setas largas en el margen posterior; meropodito casi tan largo como el propodito, con el margen posterior irregular con grupos de setas, propodito ligeramente expandido distalmente con grupos de setas en el margen posterior, subdistal y proximal, con una seta robusta subdistal y dos en la parte inferior de la palma; dactilopodito delgado, ligeramente curvado, liso, sobresaliendo ligeramente del ángulo de la palma, la palma y margen interno del dactilopodito están finamente aserradas (Fig. 2D ).

Gnatópodo 2 con coxopodito más ancho que el coxopodito 1, ventralmente triangular, artejos del basipodito al propodito similares en forma a los del gnatópodo 1, excepto por la longitud, número y posición de algunas setas, la palma y margen interno del dactilopodito están finamente aserradas (Fig. 2E).

Pereópodo 3 con coxopodito ligeramente más largo que el coxopodito 2, basipodito linear, delgado, los 2/3 proximales del margen anterior y el margen posterior con setas cortas y dispersas, carpopodito es más largo que el meropodito, con tres setas pequeñas dispersas en el margen distal anterior, meropodito y propodito subiguales, ambos delgados, dactilopodito delgado, curvado y es casi la mitad de la longitud del propodito (Fig. 3A).

Pereópodo 4 con coxopodito mucho más largo que el coxopodito 3 y es casi tan ancho como los coxopoditos 2 y 3 juntos, margen anterior sinuoso, ligeramente convexo, se curva ventralmente hacia debajo, margen posterior dividido en una profunda muesca redondeada, seguida por un proceso agudo posteroventral insertado en una abertura subcircular en el margen anterior del coxopodito 5, basipodito grueso, distalmente amplio, isquiopodito corto, carpopodito largo y delgado, meropodito más corto que el carpopodito, propodito casi tan largo como el meropodito, ligeramente curvado, dactilopodito delgado, ligeramente curvado (Fig. 3B).

Pereópodo 5 con coxopodito subtriangular, similar en tamaño al coxopodito 4, ángulo posterodistal fuertemente prolongado en forma puntiaguda, con su extremo alcanzando el primer tercio de la placa del epímero 2, basipodito el más ancho y largo de los artejos, isquiopodito corto, carpopodito, meropodito y propodito subiguales, dactilopodito curvado (Fig. 3C). Pereópodo 6 con coxopodito subcuadrado, más pequeño que el coxopodito 5 con una proyección redondeada, basipodito expandido posteriormente, isquiopodito pequeño, carpopodito, meropodito y propodito 
subiguales, dactilopodito curvado (Fig. 3D). Pereópodo 7 con coxopodito más pequeño que el coxopodito 6, basispodito expandido posteriormente, isquiopodito, meropodito carpopodito, propodito y dactilopodito similares a los del pereópodo 6 (Fig. 4A).

Urópodo 1 con el pedúnculo casi tan largo como ambas ramas, amplio distalmente, ambas ramas similares en longitud con setas cortas robustas en los márgenes (Fig. 4B). Urópodo 2 con pedúnculo más corto que ambas ramas, la rama interna es 1/5 más corta que la externa, ambas ramas con cortas setas robustas en los márgenes (Fig. 4C). Urópodo 3 con pedúnculo corto, ambas ramas similares en longitud y grosor, con cortas setas robustas en los márgenes (Fig. 4D). Epímero 1 con ángulos anteroventrales y posteroventrales ligeramente redondeados, el posteroventral es más pronunciado, epímeros $2-3$ con el ángulo anteroventral redondeado, el posteroventral pronunciado (Fig. 4F). Telson 1.2 veces tan largo como ancho, con una ligera muesca media en forma de U (Fig. 4E).

Comentarios. Epimeria panamensis sp. nov. con los caracteres presentados a continuación. Ojos ausentes, rostrum alargado y pleonitos con dientes mediodorsales. Dentro del género, solo las siguientes especies comparten esos caracteres: Epimeria morronei Winfield, Ortiz y Hendrickx, 2012 del Golfo de California, México; E. glaucosa J. L. Barnard, 1961 del Mar de Tasmania en el Océano Pacífico y E. concordia Griffiths, 1977 de Sudáfrica. Epimeria morronei tiene el coxopodito 4 con el margen anteroventral convexo, dirigido hacia atrás, margen posterior del telson aserrado y ojos vestigiales; mientras que en E. panamensis sp. nov., en el coxopodito 4 el margen anteroventral es casi recto y dirigido hacia debajo, margen posterior del telson no es aserrado y los ojos están ausentes. E. glaucosa no presenta dientes mediodorsales sobre los pereonitos, coxopodito 5 con la esquina posteroventral alargada y puntiaguda extendiéndose hasta el pleonito 1, diente mediodorsal del pleonito 1 poco desarrollado; mientras que $E$. panamensis sp. nov., presenta dientes mediodorsales en los pereionitos 5-7, coxopodito 5 con el extremo posteroventral alargado y puntiagudo extendiéndose hasta el pleonito 2 y diente mediodorsal del pleonito 1 bien desarrollado. E. concordia el rostro es largo, alcanza el final del artejo 3 de la antena 1, coxopodito 5 con el extremo posteroventral alargado y puntiagudo extendiéndose hasta el pleonito 1, segmentos del pereon lisos dorsalmente excepto por una pequeña elevación posterior en el segmento 7; E. panamensis sp. nov., presenta el rostro alargado que alcanza la mitad del artejo 2 de la antena 1, coxopodito 5 con esquina posteroventral puntiaguda extendiéndose hasta el pleonito 2, pleonitos 5-7 con dientes mediodorsales agudos dirigidos hacia detrás.

Etimología. El epíteto específico se refiere al Golfo de Panamá, lugar donde fue recolectado el espécimen.

Tipos. Holotipo. Epimeria panamensis, hembra adulta, Golfo de Panamá (Panamá), Estación (P-526) 6 51”N y 79० 28”W, 3193-3200 metros de profundidad, el 5.v.1967, con $23 \mathrm{~mm}$ de largo, UMML 32.10270. 


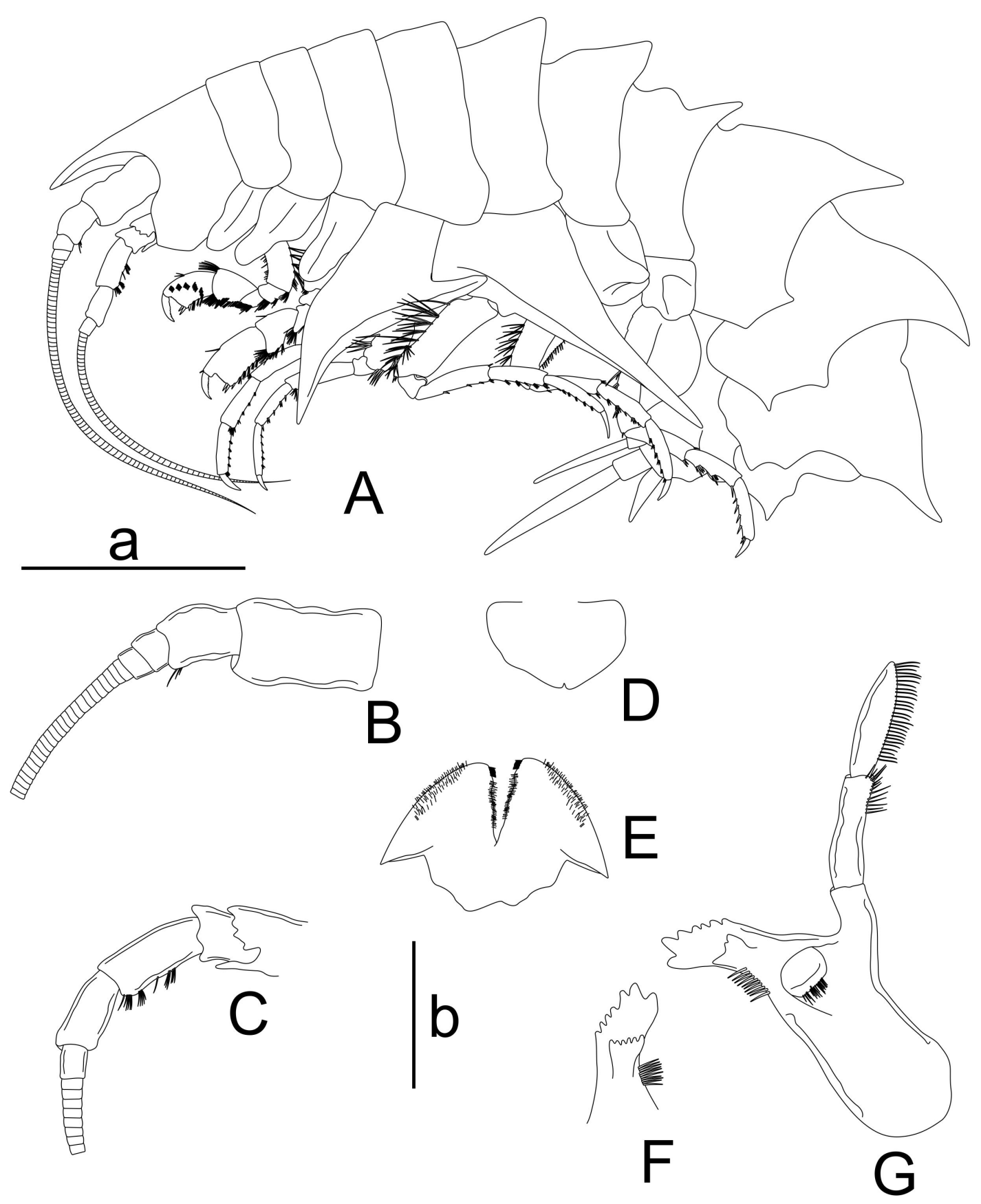

Figura 1. Epimeria panamensis sp. nov. Holotipo. A, vista lateral del ejemplar (a). B, antena 1 (b). C, antena 2 (b). D, labio superior (b). E, labio inferior (b). F, mandíbula derecha (b). G, mandíbula izquierda (b). Escalas: a, $50 \mathrm{~mm} ; \mathrm{b}, 10 \mathrm{~mm}$. 


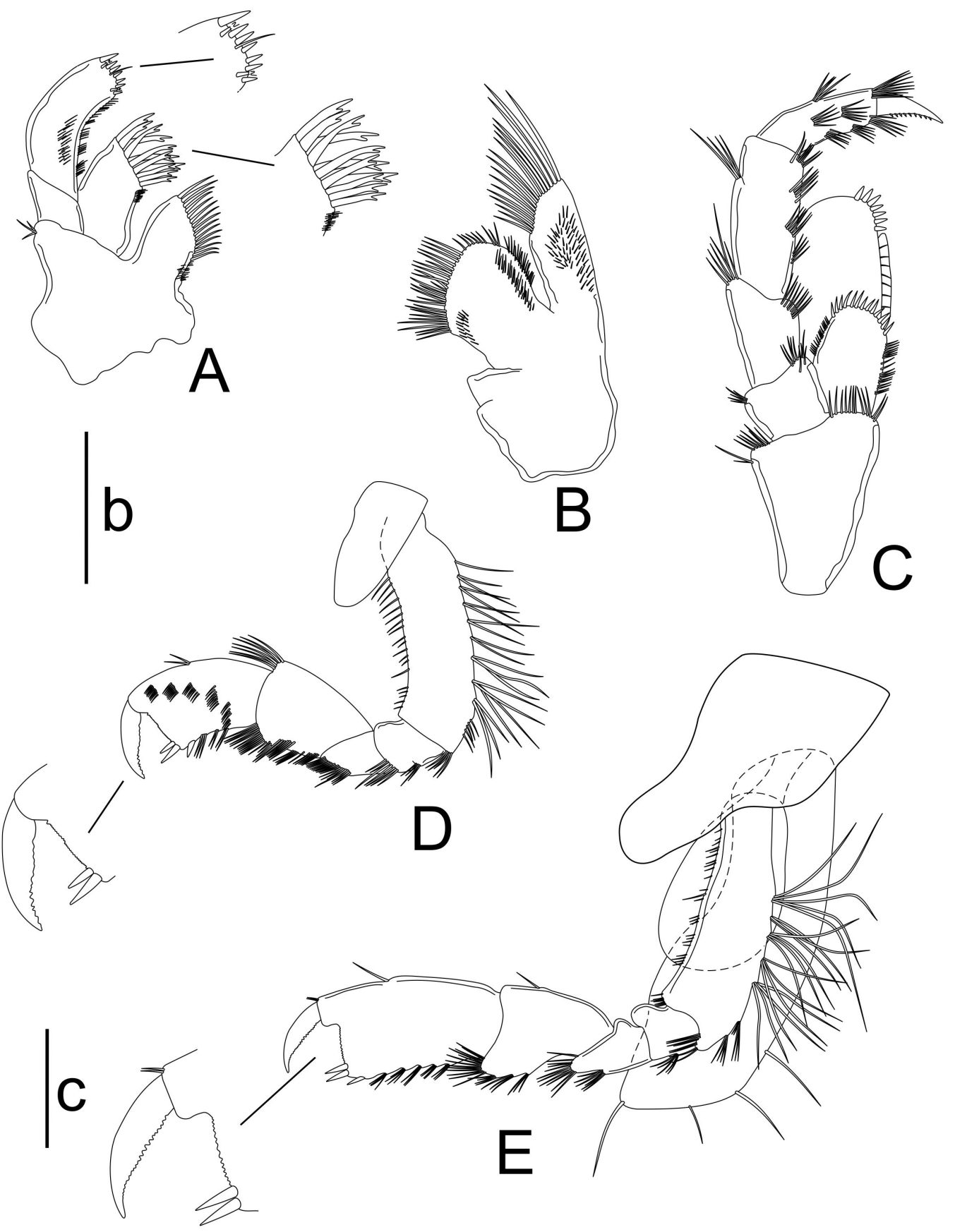

Figura 2. Epimeria panamensis sp. nov. Holotipo. A, maxila 1 (b). B, maxila 2 (b). C, maxilípedo (b). D, gnatópodo 1 (c). $\mathrm{E}$, gnatópodo 2 (c). Escalas, b y c: $10 \mathrm{~mm}$. 


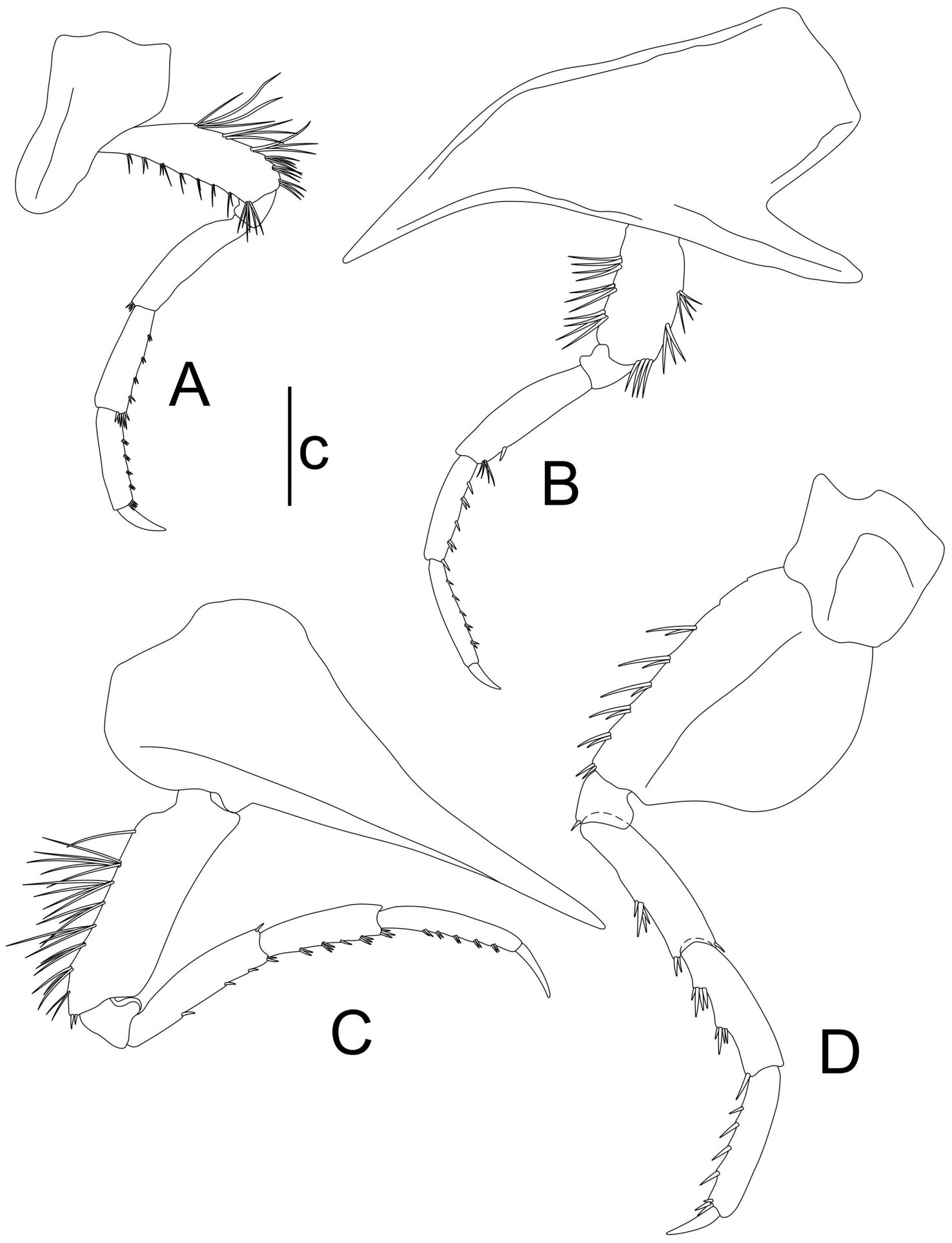

Figura 3. Epimeria panamensis sp. nov. Holotipo. A, pereópodo 3 (c). B, pereópodo 4 (c). C, pereópodo 5 (c). D, pereópodo 6 (c). Escala: c, $10 \mathrm{~mm}$. 


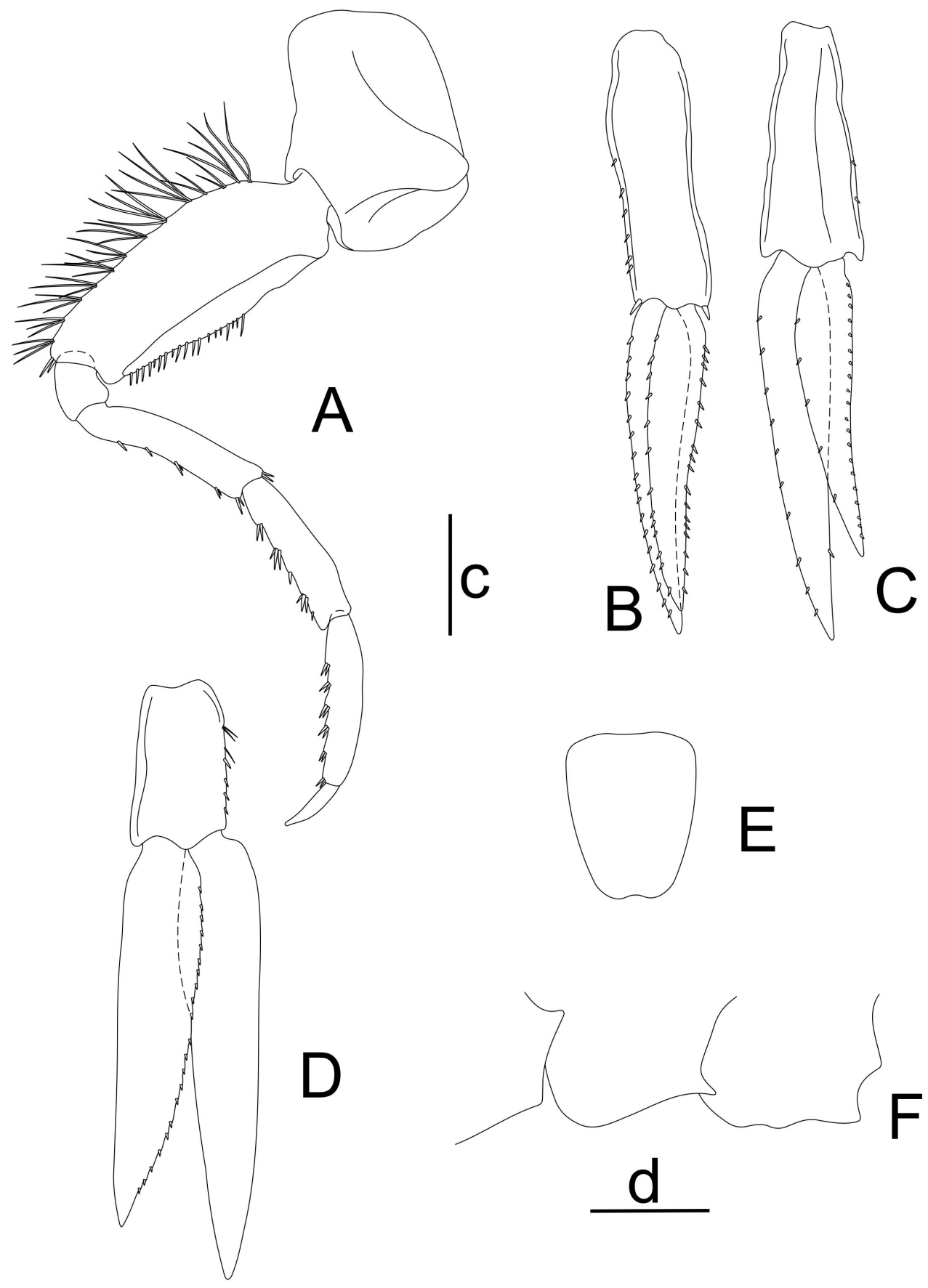

Figura 4. Epimeria panamensis sp. nov. Holotipo. A, pereópodo 7 (c). B, urópodo 1 (c). C, urópodo 2 (c). D, urópodo 3 (c). E, epímeros (d). F, telson. (c). Escalas: c y d, $10 \mathrm{~mm}$. 


\section{AGRADECIMIENTOS}

A la Research Professor Emeritus Nancy Voss, Curadora del Museo de Invertebrados Marinos de RSMAS por permitirme el estudio del material depositado en la colección. A los revisores anónimos por las correcciones al manuscrito.

\section{LITERATURA CITADA}

Beermann, J., M. V. Westbury, M. Hofreiter, L. Hilgers, F. Deister, H. Neumann y M. J. Raupach. 2018. Cryptic species in a well-known habitat: applying taxonomics to the amphipod genus Epimeria (Crustacea, Peracarida). Scientific Reports, 8 (1): 1-26.

Coleman, C. O. y J. K. Lowry. 2014. Epimeria rafaeli sp. nov. (Crustacea, Amphipoda, Epimeriidae) from Western Australia. Zootaxa, 3873 (3): 218-232.

d'Udekem d'Acoz, C. y M. L.Verheye. 2017. Epimeria of the Southern Ocean with notes on their relatives (Crustacea, Amphipoda, Eusiroidea). European Journal of Taxonomy, 359: $1-553$.

Lörz, A. y C. O. Coleman. 2014. Amazing new Amphipoda (Crustacea, Epimeriidae) from New Zealand's deep-sea. Zootaxa, 3838 (4): 423.

Lowry, J. K. y A. A. Myers. 2017. A Phylogeny and Classification of the Amphipoda with the establishment of the new order Ingolfiellida (Crustacea: Peracarida). Zootaxa, 4265 (1): 001-089.

Shimomura, M. y K. Tomikawa. 2016. Epimeria abyssalis sp. n. from the Kuril-Kamchatka Trench (Crustacea, Amphipoda, Epimeriidae). ZooKeys, 638: 125-142.

Varela, C. y J. García-Gómez. 2015. Especie nueva de Epimeria (Amphipoda: Epimeriidae) del Golfo de México y el Mar Caribe. Solenodon, 12: 1-8.

Verheye, M. L., A. N. Lörz y C. d'Udekem d'Acoz. 2018. Epimeria cleo sp. nov., a new crested amphipod from the Ross Sea, Antarctica, with notes on its phylogenetic affinities (Crustacea, Amphipoda, Eusiroidea, Epimeriidae). Zootaxa, 4369 (2): 1-186.

Winfield, I., M. Ortiz y M. E. Hendrickx. 2013. A new deep-water species of Epimeria (Amphipoda: Gammaridea: Epimeriidae) from the continental slope of western Mexico. Journal of the Biological Association of the United Kingdom, 93 (4): 991-997.

[Recibido: 19 de marzo, 2019. Aceptado para publicación: 13 de septiembre, 2019] 